\section{Revisão sistemática e meta-análise da antibioticoprofilaxia na histerectomia abdominal}

\author{
Systematic review and meta-analysis of antibiotic \\ prophylaxis in abdominal hysterectomy
}

\author{
1 Faculdade de Farmácia, \\ Universidade Federal \\ do Rio de Janeiro, \\ Rio de Janeiro, Brasil. \\ 2 Escola Nacional de Saúde \\ Pública, Fundação Oswaldo \\ Cruz, Rio de Janeiro, Brasil. \\ Correspondência \\ R. J. M. Costa \\ Departamento de Análises \\ Clínicas e Toxicológicas, \\ Faculdade de Farmácia, \\ Universidade Federal \\ do Rio de Janeiro. \\ Av. Brigadeiro Trompowsky \\ $s / n$, Centro de Ciências \\ da Saúde, Bloco A, \\ 2 o andar, sala 47 , \\ Rio de Janeiro, $R J$ \\ 21941-590, Brasil. \\ justen@pharma.ufrj.br
}

\begin{abstract}
The objective of the present study was to assess the available evidence regarding antibiotic prophylaxis taking the case of abdominal hysterectomy, as an aid to decisions related to coverage and to the development of evidence-based clinical guidelines. Using a previously elaborated protocol, the pertinence and quality of doubleblind, randomized, placebo-controlled trials were examined. Clinical heterogeneity among studies was also analyzed. The studies were found to be homogeneous, considering $p>0.10$ as the significance level for rejecting heterogeneity. Combination of the 16 selected studies resulted in a summary rate ratio of 0.49 (95\%CI: 0.41-0.59), i.e., efficacy of 51\%, and in a summary rate difference of $11 \%$ (95\%CI: 8-14), utilizing the fixed effects model. Results according to the random effects model were very similar. Exploratory analysis of subgroups of single versus multiple doses did not indicate more benefit for multiple dose interventions. Evidence from comparative trials is insufficient to consider thirdgeneration cephalosporins more efficacious than first-generation ones.
\end{abstract}

Meta-Analysis; Antibiotic Prophylaxis; Hysterectomy; Evidence-Based Medicine
Ricardo Justen Moreira da Costa 1 Letícia Krauss-Silva 2

\section{Introdução/justificativa}

A infecção pós-cirúrgica é um problema relevante de saúde. Nos Estados Unidos, estima-se que dos 23 milhões de cirurgias realizadas por ano, 920 mil são complicadas por infecções da ferida operatória, o que está associado ao aumento do tempo de internação, das taxas de letalidade e dos gastos hospitalares 1. No Brasil, Ferraz et al. 2 encontraram uma taxa média de infecção da ferida cirúrgica próxima a $10 \%$, com base em um estudo prospectivo efetuado em um hospital universitário entre 1995 e 1999.

De acordo com as diretrizes do Ministério da Saúde 3 sobre o assunto, o uso de antimicrobianos pode prevenir a ocorrência de infecção pós-operatória no sítio cirúrgico em vários tipos de cirurgias, inclusive as histerectomias. Porém, informações e referências relativas ao exame das evidências científicas correspondentes não são mencionadas pelo documento. Além disso, as diretrizes oferecidas não são claras quanto ao esquema preventivo a ser utilizado.

A revisão sistemática busca sintetizar resultados de estudos sobre benefícios e efeitos adversos de tecnologias baseando-se em estudos disponíveis de boa qualidade, constituindo-se em uma metodologia importante para a elaboração de uma avaliação tecnológica. Utiliza uma abordagem sistemática que objetiva evitar que seja distorcido o tamanho do efeito estudado, provendo uma base científica para a 
tomada de decisão não só de clínicos, mas de planejadores e gerentes. A meta-análise é uma forma de revisão sistemática na qual é efetuada uma combinação quantitativa dos resultados de vários estudos, para a obtenção de uma estimativa única do resultado, ou seja, uma medida sumária do(s) efeito(s) analisado(s) 4,5.

O presente estudo teve como objetivo examinar a evidência científica disponível relativa à eficácia da antibioticoprofilaxia cirúrgica no caso da histerectomia, a segunda cirurgia ginecológica em termos de freqüência no Sistema Único de Saúde, com cerca de 80 mil cirurgias em 20026 , no sentido de subsidiar decisões relativas ao financiamento desse procedimento e contribuir para a produção de diretrizes clínicas baseadas em evidências, no que diz respeito à sua indicação e esquema profilático a ser utilizado.

\section{Metodologia}

\section{Estudo dos fatores prognósticos} relativos à infecção pós-cirúrgica

No intuito de subsidiar a análise da qualidade e da heterogeneidade clínica dos ensaios sobre a antibioticoprofilaxia, procedemos o exame da evidência sobre fatores prognósticos de infecção pós-cirúrgica, a seguir.

Os estudos prospectivos disponíveis com análises multivariadas sobre fatores prognósticos para infecção do sítio cirúrgico em geral, identificados após consulta às bases de dados MEDLINE, LILACS e Web of Sciences, indicam que a classificação tradicional das feridas (limpa, limpa-contaminada, contaminada e infectada) da Academia Nacional de Ciências dos Estados Unidos 7 é insuficiente para discriminar os pacientes por grupos de risco de infecção. Além do tipo de ferida (odds ratio $[\mathrm{OR}]=2,7$; intervalo de confiança [IC95\%]: 1,9-4,6), os fatores $d u$ ração da cirurgia $>2$ horas (OR = 3,0; IC95\%: $1,6-3,6) 8$ e estado geral do paciente, indicado pelo número de comorbidades (coeficiente de regressão $=0,86 ; p<0,0001$ ) 9 , ou pelo escore de gravidade da American Society of Anesthesiologists - ASA (OR = 2,4; IC95\%: 1,8-4,0) 8 parecem ter também grande importância prognóstica.

O exame de estudos disponíveis prospectivos, com análises uni e multivariadas sobre fatores prognósticos de infecção em histerectomias 10,11,12, confirma a duração da cirurgia $>2$ horas como fator prognóstico relevante e identifica o fator via cirúrgica abdominal.

Segundo a referida classificação da ferida operatória, a histerectomia abdominal pertence à categoria "limpa", desde que o procedi- mento seja eletivo, e não exista inflamação aguda no campo operatório. Quanto à duração da cirurgia, a histerectomia abdominal eletiva pode ser caracterizada como um procedimento com duração próxima a 2 horas, isto é, um procedimento de baixo risco para infecção pósoperatória quanto ao tempo cirúrgico 13.

Considerando que os subtipos e as abordagens para a histerectomia em geral são heterogêneas do ponto de vista de fatores prognósticos para infecção pós-cirúrgica 14 , optamos por analisar a evidência relativa ao subtipo abdominal eletiva. Este é o subtipo de histerectomia mais realizado em nosso país 6 .

\section{Evidências sobre efeitos}

\section{da antibioticoprofilaxia a partir} de modelos animais

Burke 15 submeteu porquinhos-da-índia à infecção experimental subcutânea com bactérias, sendo mostrado graficamente que a administração do antimicrobiano dentro de uma hora antes da inoculação reduziu acentuadamente o tamanho das lesões resultantes, frente àquelas do grupo controle. Vinte anos depois, Shapiro et al. 16 confirmaram os achados de Burke, observando uma redução das lesões de cerca de $50 \%(\mathrm{p}<0,001)$.

O modelo de Burke indicou que para exercer ação profilática, o antimicrobiano deve estar presente nos tecidos no momento da incisão e da contaminação bacteriana do sítio operatório. Além de Burke, dois estudos bem desenhados, em humanos, apontam também a importância da cobertura antimicrobiana da janela cirúrgica 17,18 .

\section{Revisão das evidências científicas} sobre efeitos da antibioticoprofilaxia cirúrgica relativas à histerectomia abdominal eletiva, não radical

\section{- Identificação e seleção de revisões sistemáticas e meta-análises disponíveis}

A partir da estratégia de busca que incluiu pesquisas efetuadas nas bases de dados MEDLINE, 1966-2003, Web of Sciences, 1966-2002 e Cochrane Library, até 2003, foram identificadas quatro revisões sistemáticas e meta-análises de antibioticoprofilaxia em histerectomia abdominal eletiva 19,20,21,22. O efeito por elas investigado foi o da eficácia. Passou-se, então, ao exame da qualidade do desenho e execução, bem como da atualidade desses estudos.

Quase todas as meta-análises admitiram apenas ensaios clínicos randomizados e con- 
trolados por placebo. Mas somente Polk 19 relatou como critério a seleção de ensaios duplamente cegados. Não identificamos meta-análises de ensaios comparativos de modalidades de intervenção (antibioticoprofilaxia). As quatro meta-análises apresentaram resultados sobre infecção do sítio operatório, que incluíram os desfechos (end-points) infecção da ferida cirúrgica e infecção da região pélvica. Mas em nenhuma delas os critérios para aqueles desfechos foram delimitados.

Também não foi encontrada qualquer referência à ocorrência de perdas ou intervenções pós-randomização nos ensaios, nem a explicitação do tipo de análise por eles realizada: segundo intenção de tratar e/ou segundo tratamento efetivo 23 . Os estudos também não referem exame das fontes de heterogeneidade clínica, que são possíveis fontes de heterogeneidade de resultados (eficácia), embora duas meta-análises 20,21 tenham realizado testes de heterogeneidade estatística.

A combinação dos ensaios feita pelas metaanálises citadas resultou em estimativas de efeito sumário relativamente semelhantes, com os riscos relativos (RR) variando entre 0,43 e 0,50 (isto é, entre $57 \%$ e $50 \%$ de eficácia) e as diferenças de riscos (DR) entre $7 \%$ e $12 \%$ para infecção do sítio operatório na histerectomia abdominal eletiva. Não são mencionadas análises de sensibilidade, relacionadas à incerteza sobre ou à ocorrência de falhas metodológicas.

Apesar da consistência dos referidos RR, a limitação das informações fornecidas pelas meta-análises quanto à sua consecução e sobre as características dos ensaios por elas analisados, apontaram a necessidade de uma análise mais detalhada dos ensaios clínicos disponíveis para eventual meta-análise de ensaios pertinentes e de qualidade satisfatória.

\section{- Elaboração de nova revisão sistemática}

Buscou-se então analisar a pertinência/validade para os objetivos do presente estudo e a qualidade do desenho, da execução e da análise de dados feitas por ensaios, enquanto possíveis fatores que podem distorcer o tamanho do efeito da antibioticoprofilaxia para a cirurgia estudada. Procurou-se também examinar outra fonte de heterogeneidade de resultados, aquela derivada de fatores clínicos, ou seja, a derivada: (a) da estrutura de fatores prognósticos (enquanto confundidores e possíveis modificadores de efeito); (b) dos esquemas terapêuticos e (c) das definições de desfechos (end-points), no sentido de avaliar a validade de efetuar a meta-análise 24,25. Esse esforço teve o propósito de não apenas analisar com maior cuidado a validade das conclusões das meta-análises já disponíveis, mas também procurar fornecer subsídios mais detalhados à elaboração de diretrizes clínicas aplicáveis à nossa realidade.

\section{a) Identificação e seleção de ensaios clínicos}

Para proceder a análise sistemática e detalhada da evidência disponível sobre os efeitos da antibioticoprofilaxia na histerectomia abdominal eletiva, foi utilizada uma estratégia de busca à literatura para identificação de ensaios clínicos, que incluiu pesquisas efetuadas nas bases de dados MEDLINE, 1966-2003, Web of Sciences, 1966-2002, LILACS, até 2003 e Cochrane Library, até 2003 (que inclui ensaios citados pela Embase), sem restrição quanto ao idioma empregado. As palavras truncadas (wild-cards) utilizadas foram: hysterectom, antimicrobia, antibioti, prophyla, chemoprophyla, surger, e operatio. Também foram consultadas as referências bibliográficas das referidas meta-análises e dos ensaios clínicos, além das citadas em capítulos de dois livros-textos relativos ao assunto: Mead 14 e Clarke-Pearson et al. 26. Esta estratégia foi efetuada com base na aplicação dos princípios da estratégia de busca de alta sensibilidade, para a localização de ensaios clínicos por meio do MEDLINE sugerida por Dickersin et al. 27 e pela Cochrane Collaboration 28 , e por intermédio do LILACS conforme Castro et al. 29 e o Centro Cochrane do Brasil (http://www.cen trocochranedobrasil.org.br/lilacs.asp, acessado em 21/Mai/2004). A partir dessa busca, foram encontrados 138 ensaios clínicos controlados sobre o assunto.

De acordo com o protocolo da presente revisão, foram considerados os seguintes critérios para inclusão de ensaios clínicos: (a) ser um estudo randomizado e duplo-cego; (b) apresentar comparação entre grupo(s) submetido(s) à antibioticoprofilaxia e grupo controlado por placebo ou comparativo em pacientes submetidas à histerectomia abdominal eletiva, não radical; (c) ter iniciado a profilaxia pela via parenteral até 2 horas antes do início da cirurgia; (d) ter como desfecho geral a infecção do sítio operatório, incluindo os casos relativos aos subtipos de infecção da ferida operatória (superficial e profunda) e de infecção pélvica (intra cavitária), tomando-se por base as definições do Centers for Disease Control and Prevention (CDC) 30,31 (Tabela 1).

A existência de ensaios com duração da profilaxia superior a 24 horas, chegando até nove dias, o que corresponde mais a tratamento do que a antibioticoprofilaxia cirúrgica, fez com 
Critérios do Centers for Disease Control and Prevention (CDC) para infecção pós-cirúrgica (Horan et al. 30 basicamente ratificados por Mangram et al. ${ }^{31}$ ).

Infecção superficial incisional

Infecção incisional profunda

Infecção intracavitária

Condições gerais
Envolve somente a pele e/ou tecido subcutâneo da incisão, com pelo menos um dos seguintes achados: (a) secreção purulenta; (b) organismos isolados a partir de cultura; (c) abertura da incisão superficial pelo cirurgião acompanhada de pelo menos um dos seguintes sinais ou sintomas: dor ou brandura, edema, hiperemia ou calor localizados; (d) diagnóstico efetuado pelo cirurgião ou médico.

Envolve tecidos profundos da incisão (isto é, fáscia e músculos), com pelo menos um dos seguintes achados: (a) secreção purulenta oriunda do tecido incisional profundo; (b) deiscência ou abertura pelo cirurgião da incisão profunda, acompanhada de pelo menos um dos seguintes sinais ou sintomas: febre $>38 \circ \mathrm{C}$, dor ou brandura; (c) abscesso ou outra evidência de infecção, observada diretamente (reoperação) ou através de exame por imagem ou histopatológico, na região incisional profunda; (d) diagnóstico efetuado pelo cirurgião ou médico.

Envolve órgão ou cavidade aberta ou manipulada durante a cirurgia, menos a incisão, e pelo menos um dos seguintes achados: (a) drenagem de pus oriundo da cavidade; (b) organismos isolados a partir de cultura; (c) abscesso ou outra evidência de infecção, observada diretamente (reoperação) ou por meio de exame por imagem ou histopatológico, envolvendo a cavidade ou órgão; (d) diagnóstico efetuado pelo cirurgião ou médico.

Ocorrência dentro de trinta dias; cultura de fluido ou tecido assepticamente colhido do subsítio de infecção presumida; tanto o diagnóstico de infecção quanto a abertura da incisão feitos pelo cirurgião são critérios de infecção desde que a cultura ou outro exame não forneça evidência em contrário. que determinássemos o seguinte critério adicional de inclusão de ensaios: (e) ter um curso de antibioticoprofilaxia de, no máximo, 24 horas.

Com base nos critérios acima, restaram 16 ensaios placebo-controlados, que totalizaram vinte comparações entre profilaxia versus placebo. Dentre esses ensaios não foram identificados estudos comparativos de dose única versus múltiplas doses do mesmo antimicrobiano. Foi então prevista a análise exploratória da eficácia de ensaios de dose única versus aquela de ensaios de múltiplas doses. Análise semelhante foi prevista considerando os subgrupos de ensaios com cobertura antibiótica pela concentração inibitória mínima (CIM) por até três horas (perioperatório) e por mais de três horas. Nesse caso, objetivou-se obter uma estimativa mais acurada do tempo de cobertura antimicrobiana que aquela indicada pelo número/intervalo de doses.

Dentre os 138 ensaios resultantes da estratégia de busca encontramos estudos comparativos que, em sua maioria, não focalizavam comparações de interesse, isto é, que compreendessem drogas com diferenças de espectro de ação relevantes e esquemas de curta duração. Apenas três ensaios abrangiam comparações relevantes. Dois deles 32,33 apresentavam problemas metodológicos, inclusive ausência de cegamento. Apenas o estudo de Hemsell et al. 34 apresentou qualidade satisfatória, comparando cefalosporinas de 1a e $3 \underline{a}$ gerações em regimes de dose única, e encontrando para estas últimas eficácia marginalmente superior: $\mathrm{RR}=$ 0,51; IC95\%: 0,28-0,92; $\mathrm{p}=0,027$.

\section{b) Análise mais detalhada da qualidade dos ensaios clínicos placebo-controlados}

A revisão sistemática dos ensaios foi feita em duas etapas independentes, a primeira relativa à metodologia e execução do ensaio e a segunda relativa aos resultados, com base em questionário/grade preparado a partir do protocolo. Em todos os ensaios foi referida a realização de randomização, porém não houve, em geral, relato do método utilizado ou menção de fatores de estratificação. A maioria dos ensaios também não informou sobre ocultação do esquema de alocação. Embora a grande maioria tenha apresentado uma tabela com a distribuição das características de base (tabela pós-randomização), a distribuição dos fatores prognósticos analisados não incluiu, em geral, aqueles apontados pelos estudos bem desenhados, anteriormente revisados (duração da cirurgia e comorbidade/estado geral das pacientes). Tais falhas limitaram o exame da utilização e do sucesso de procedimentos para evitar a ocorrência de viés de seleção de pacientes nos ensaios.

Dos ensaios selecionados, duplo-cegos, metade informou ainda que os cirurgiões foram cegados, embora apenas $25 \%$ dos ensaios tenham explicitado o procedimento de cegamento utilizado. 
Quanto à execução do ensaio e à análise de dados, a maior parte dos estudos não forneceu informações sobre perdas ou intervenções pósrandomização, nem referiu ou deixou implícito se a análise realizada foi segundo intenção de tratar ou segundo tratamento efetivo. Alguns ensaios 35,36,37 informaram a ocorrência de um pequeno número de perdas. Outros mencionaram perdas importantes, entre 10 e $20 \%$ das pacientes após a randomização, não incluídas na análise, e em geral balanceadas entre os grupos de tratamento $38,39,40$. As perdas foram devidas em grande parte ao tratamento antimicrobiano em função do prolongamento ou complicação da cirurgia, e também aos dados/prontuários perdidos.

Além das fontes de heterogeneidade de resultados analisadas acima (desenho, execução e análise de dados dos ensaios), tem-se ainda a $h e$ terogeneidade clínica, que passamos a analisar.

\section{c) Análise da heterogeneidade clínica entre os ensaios}

(i) Quanto à estrutura de fatores prognósticos dos pacientes: a maioria dos ensaios utilizou como critérios de exclusão apenas a evidência de infecção na admissão e/ou uso de antimicrobiano para tratamento de infecção nos dias que antecederam a cirurgia, além de história de alergia ao antimicrobiano testado. Conforme já comentado, os ensaios não apresentaram, em geral, informações sobre a estrutura dos fatores prognósticos apontados anteriormente, o que limitou também a avaliação da heterogeneidade entre ensaios nesse aspecto.

(ii) Quanto ao tipo de intervenção antimicrobiana realizada: a análise mais detalhada dos ensaios mostrou existir uma grande diversidade de intervenções, sobretudo quanto ao antimicrobiano empregado e quanto à duração da profilaxia. De um modo geral, as intervenções estudadas eram válidas em tese do ponto de vista do potencial de eficácia e segurança. Os antimicrobianos mais testados pelos ensaios foram as cefalosporinas, os aminoglicosídeos e alguns análogos de penicilinas, correspondendo a uma heterogeneidade em termos de espectro de ação, o que pode ser uma fonte de heterogeneidade de resultados dos ensaios para os desfechos analisados.

Ainda com relação ao esquema profilático, observou-se heterogeneidade entre os ensaios quanto à dose, ao número de doses e à duração dos esquemas utilizados, outras possíveis fontes de heterogeneidade de resultados.

(iii)Quanto aos desfechos (end-points) medidos pelos ensaios: a maioria dos ensaios defi- niu alguns dos desfechos, infecção da ferida operatória ou infecção pélvica, utilizando critérios abrangidos pelas definições do CDC (Tabela 1) 30,31. No entanto, houve diferença entre os estudos quanto à sensibilidade do critério diagnóstico, o que poderia propiciar diferenças nos resultados dos ensaios. Também o tempo de seguimento variou entre os estudos desde o período de hospitalização (cerca de uma semana) até seis semanas.

\section{d) Técnicas analíticas empregadas para a estimativa da eficácia}

Os dados dos ensaios foram extraídos e analisados utilizando-se o software estatístico Metaanalyst versão 0,991 41. Foram então calculados os RR e os IC, com nível de confiança de 95\%, primeiramente para cada ensaio e depois para a combinação dos ensaios, por meio dos modelos de efeitos fixos (MEF) e de efeitos aleatórios (MEA). Utilizou-se o teste do $\chi^{2}$ para o cálculo da heterogeneidade estatística, considerandose o valor de $\mathrm{p}>0,1$ como nível de significância para aceitar que os resultados são homogêneos.

\section{Resultados (estimativa da eficácia)}

Os RR e os respectivos IC95\% dos 16 estudos selecionados e os relativos à medida sumária para o desfecho geral, infecção do sítio cirúrgico, são mostrados na Tabela 2 e na Figura 1. O teste de heterogeneidade estatística, a despeito da variação dos resultados dos ensaios individuais, indicou homogeneidade $(\mathrm{p}>0,10)$. A combinação dos 16 ensaios apontou a existência de benefício clinicamente relevante da antibioticoprofilaxia cirúrgica na prevenção da infecção em geral do sítio operatório, após a cirurgia de histerectomia abdominal eletiva, não radical: RR sumário de 0,49 (IC95\%: 0,41-0,59), ou seja, eficácia de $51 \%$ com DR de $11 \%$ (IC95\%: 8-14\%), utilizando o modelo de efeitos fixos, com resultados muito semelhantes aos observados usando-se o modelo de efeitos aleatórios.

A análise exploratória do efeito de ensaios de dose única em comparação com o de múltiplas doses, Tabela 3, mostrou eficácia preventiva importante da infecção do sítio operatório, de cerca de $50 \%$, do regime de dose única: $\mathrm{RR}=$ 0,53 (IC95\%: 0,34-0,79); DR = 14\% (IC95\%: 8$19 \%$ ) / MEF. Esses resultados são muito semelhantes aos do conjunto de ensaios e aos obtidos a partir da combinação daqueles ensaios que utilizaram regimes de múltiplas doses: $\mathrm{RR}=$ 0,48 (IC95\%: 0,38-0,60); DR = 10\% (IC95\%: 7 $13 \%$ ) / MEF. O intervalo de confiança do grupo 
Taxas de infecção do sítio cirúrgico em ensaios clínicos placebo-controlados de antibioticoprofilaxia na histerectomia abdominal eletiva, não radical.

\begin{tabular}{|c|c|c|c|c|}
\hline $\begin{array}{l}\text { Ensaios selecionados } \\
\text { (1ㅇa autor) }\end{array}$ & $\begin{array}{c}\text { Número de pacientes } \\
\text { (placebo } x \text { antimicrobiano) }\end{array}$ & \multicolumn{2}{|c|}{ Infecção do sítio operatório (\%) } & RR (IC95\%) \\
\hline Mathews 46 & $29 \times 30$ & $11(38 \%)$ & $8(26 \%)$ & $0,70(0,33-1,49)$ \\
\hline Holman 47 & $38 \times 42$ & $13(34 \%)$ & $2(5 \%)$ & $0,14(0,03-0,58)$ \\
\hline Roberts 48 & $22 \times 25$ & $3(14 \%)$ & $1(4 \%)$ & $0,29(0,03-2,62)$ \\
\hline Polk 40 & $223 \times 203$ & 47 (21\%) & $29(14 \%)$ & $0,68(0,44-1,03)$ \\
\hline Itskovitz 35 & $36 \times 33$ & $10(28 \%)$ & $1(3 \%)$ & $0,11(0,01-1,81)$ \\
\hline Stage 49 & $42 \times 68$ & $2(5 \%)$ & $1(1,5 \%)$ & $0,31(0,03-3,30)$ \\
\hline Duff 50 & $46 \times 45$ & $12(27 \%)$ & $8(18 \%)$ & $0,68(0,31-1,51)$ \\
\hline Vicelette 36 & $53 \times 53$ & $7(13 \%)$ & $7(13 \%)$ & $1,00(0,38-2,65)$ \\
\hline Hemsell 7 & $50 \times 50$ & $16(32 \%)$ & $6(12 \%)$ & $0,38(0,16-0,88)$ \\
\hline Gall 51 & $17 \times 22$ & $5(23 \%)$ & $0(0 \%)$ & $0,07(0,00-1,20)$ \\
\hline Gall 51 & $17 \times 19$ & $5(23 \%)$ & $0(0 \%)$ & $0,08(0,00-1,38)$ \\
\hline Houang 39 & $49 \times 51$ & $12(24 \%)$ & $2(4 \%)$ & $0,16(0,04-0,68)$ \\
\hline Houang 39 & $49 \times 58$ & $12(24 \%)$ & $2(3 \%)$ & $0,14(0,03-0,60)$ \\
\hline Gonen 52 & $53 \times 63$ & $20(38 \%)$ & $9(14 \%)$ & $0,38(0,19-0,76)$ \\
\hline Evaldson 37 & $49 \times 49$ & $11(22 \%)$ & $5(10 \%)$ & $0,45(0,17-1,21)$ \\
\hline Hakim 53 & $53 \times 52$ & $20(38 \%)$ & $12(23 \%)$ & $0,61(0,33-1,12)$ \\
\hline Hakim 53 & $53 \times 54$ & $20(38 \%)$ & $10(18 \%)$ & $0,49(0,25-0,95)$ \\
\hline Davey 42 & $102 \times 97$ & $9(28 \%)$ & $16(16 \%)$ & $0,58(0,34-1,00)$ \\
\hline Davey 42 & $102 \times 101$ & $29(28 \%)$ & $22(22 \%)$ & $0,77(0,47-1,24)$ \\
\hline Henriksson 38 & $124 \times 134$ & $16(13 \%)$ & $8(6 \%)$ & $0,46(0,21-1,04)$ \\
\hline Total & $1.207 \times 1.249$ & $300(25 \%)$ & $149(12 \%)$ & - \\
\hline \multicolumn{5}{|c|}{ Teste de heterogeneidade: $\chi^{2}=23,92, p=0,199$} \\
\hline \multicolumn{2}{|c|}{ Medida sumária: modelo de efeitos fixos } & \multicolumn{2}{|c|}{$R R=0,49 ;$ IC95\%: 0,41-0,59 } & \\
\hline \multicolumn{2}{|l|}{ Mantel \& Haenszel } & \multicolumn{2}{|c|}{$D R=11 \% ; I C 95 \%: 8-14 \%$} & \\
\hline \multicolumn{2}{|c|}{ Medida sumária: modelo de efeitos aleatórios } & \multicolumn{2}{|c|}{$R R=0,51 ; I C 95 \%: 0,41-0,63$} & \\
\hline \multicolumn{2}{|l|}{ DerSimonian \& Laird } & \multicolumn{2}{|c|}{$D R=13 \% ; I C 95 \%: 9-17 \%$} & \\
\hline
\end{tabular}

$\mathrm{RR}=$ risco relativo; $\mathrm{IC95 \%}=$ intervalo de confiança de 95\%; $\mathrm{DR}=$ diferença de risco.

de dose única é, entretanto, mais amplo, em boa parte refletindo o menor número de pacientes envolvidos.

A análise exploratória segundo tempo de cobertura pela CIM mostrou as seguintes medidas sumárias: (a) subgrupo com CIM $\leq 3$ horas (dois ensaios: Houang et al. 39 - braço amoxacilina + sulbactam, e Davey et al. 42 , ambos os braços): $\mathrm{RR}=0,58$ (IC95\%: 0,41-0,82); $\mathrm{DR}=$ 12\% (IC95\%: 6-19\%), resultado um pouco menos favorável que os anteriores e apresentando IC95\% mais amplo; (b) subgrupo com CIM > 3 horas (demais ensaios): $\mathrm{RR}=0,46$ (IC95\%: 0,370,57); DR $=12 \%$ (IC95\%: 6-19\%), resultado semelhante ao original.

Tendo em vista a grande diversidade de regimes antimicrobianos utilizados pelos ensaios placebo-controlados, analisamos exploratoriamente o benefício de antimicrobianos de espectros mais e menos restritos (Tabela 4).
O agrupamento de ensaios que empregaram cefalosporinas e análogos de penicilina resultou em medida sumária (Tabela 4) bastante semelhante à original: RR de 0,48 (IC95\%: 0,360,61), com DR de 15\% (IC95\%: 9-16\%)/MEF. Por outro lado, a combinação dos estudos que utilizaram drogas com cobertura restrita aos microorganismos anaeróbios (Tabela 4) resultou em medidas sumárias menos favoráveis, com intervalos de confiança que se aproximam do efeito nulo: $\mathrm{RR}=0,60$ (IC95\%: 0,39-0,93); $\mathrm{DR}=$ 7\% (IC95\%: 1-12\%) /MEF.

Considerando a diferença na estrutura de bactérias associadas à infecção da ferida e à infecção pélvica e a diferença de espectro dos antibióticos estudados, analisamos ainda o resultado dos ensaios por subtipo de infecção do sítio cirúrgico (Tabela 5).

Os efeitos sumários e intervalos de confiança são praticamente idênticos para os dois des- 
Taxas de infecção do sítio cirúrgico em ensaios placebo-controlados de antibioticoprofilaxia na histerectomia abdominal eletiva, não radical. Modelo de efeitos fixos (Mantel \& Haenszel).

Risco relativo e intervalo de confiança $95 \%$

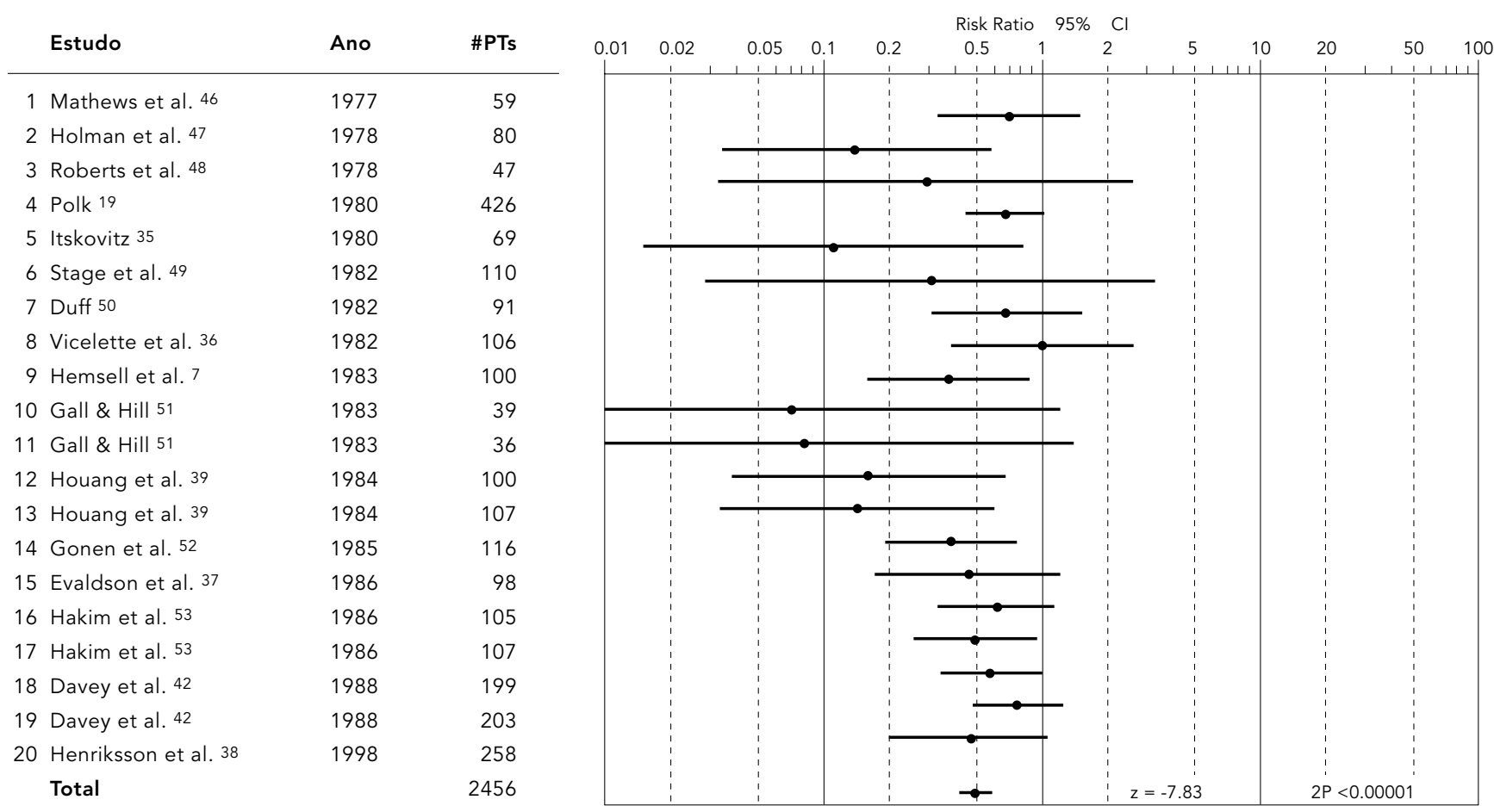

Heterogeneidade: $\chi^{2}=23,92 ; \mathrm{p}=0,199$

$\mathrm{RR}=0,49$; IC95\%: 0,41-0,59

DR: $11 \%$; IC95\%: 8-14\%

fechos. A análise dos ensaios individualmente segundo desfecho também mostra, em geral, benefício para a antibioticoprofilaxia para ambos os desfechos. A análise de sensibilidade com exclusão do ensaio que não informou os resultados relativos aos subtipos de infecção 42 , registrando apenas seu somatório, não mudou o resultado da meta-análise.

Com base nos resultados de RR dos ensaios placebo-controlados, foi construído um gráfico de funil no intuito de avaliar a existência de viés de publicação ${ }^{43}$. Os resultados revelaram a existência de grande assimetria devido à ausência de ensaios negativos.

\section{Discussão}

O objetivo do presente estudo foi analisar a evidência científica disponível sobre a eficácia da antibioticoprofilaxia no caso da histerectomia abdominal eletiva, no sentido de subsidiar as decisões relativas ao financiamento e a elaboração das diretrizes clínicas correspondentes. Esse esforço focalizou principalmente a análise detalhada da qualidade/validade dos ensaios e das fontes de heterogeneidade dos resultados, aspectos pouco abordados nas revisões sistemáticas disponíveis sobre o assunto, buscando adequadamente esclarecer se, em que momento, por quanto tempo (número de doses) e quais antimicrobianos deveriam ser utilizados para evitar infecção no sítio cirúrgico na histerectomia abdominal eletiva.

Quanto à existência de viés de publicação, o gráfico de funil revelou uma grande assimetria. Esse aspecto aponta a possibilidade de que estudos com resultados negativos, principalmente de pequeno porte, não tenham sido publicados, o que implicaria a superestimativa 
Taxas de infecção do sítio cirúrgico em ensaios clínicos placebo-controlados de antibioticoprofilaxia na histerectomia abdominal eletiva, não radical

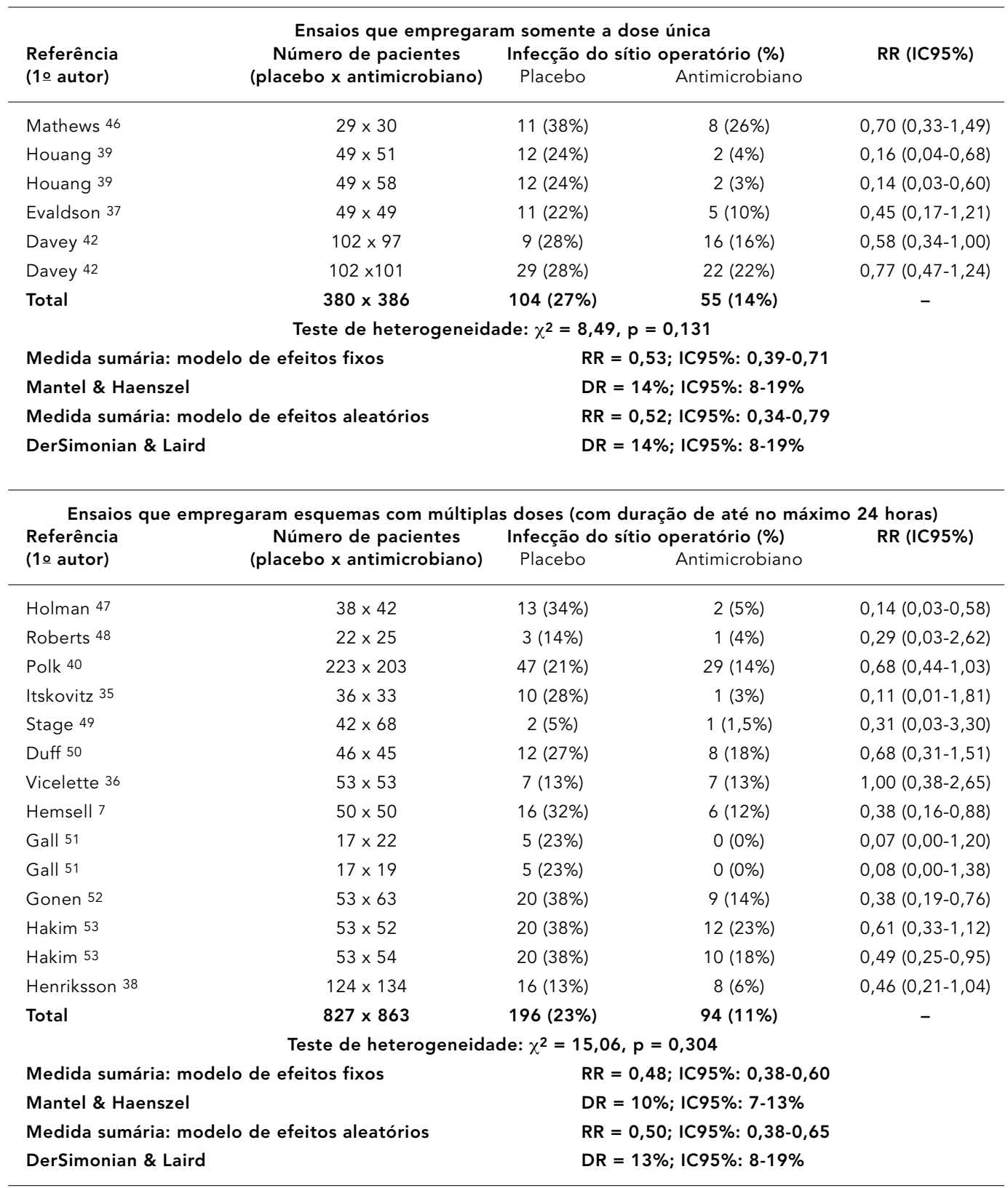

$\mathrm{RR}=$ risco relativo; $\mathrm{IC95 \%}=$ intervalo de confiança de 95\%; $\mathrm{DR}=$ diferença de risco.

do efeito estudado pela meta-análise. Outras explicações para a assimetria poderiam ser falhas metodológicas e diferença de prognóstico dos pacientes dos pequenos ensaios 44 .

Todos os ensaios selecionados se referiram como duplo-cegos, metade deles informou ainda que também os cirurgiões teriam sido cegados, mas apenas um quarto explicitou os procedimentos efetivamente realizados para tal.
Esses ensaios referiram procedimento que também deu conta da ocultação do esquema de alocação, informando que o código relativo ao tratamento destinado a cada paciente teria sido utilizado apenas pelos profissionais da Farmácia, encarregados da dispensação do antimicrobiano e do placebo. Por outro lado, conforme explicitado em alguns ensaios, normalmente é o próprio cirurgião o profissional en- 
Taxas de infecção do sítio cirúrgico em ensaios clínicos placebo-controlados de antibioticoprofilaxia na histerectomia abdominal eletiva, não radical.

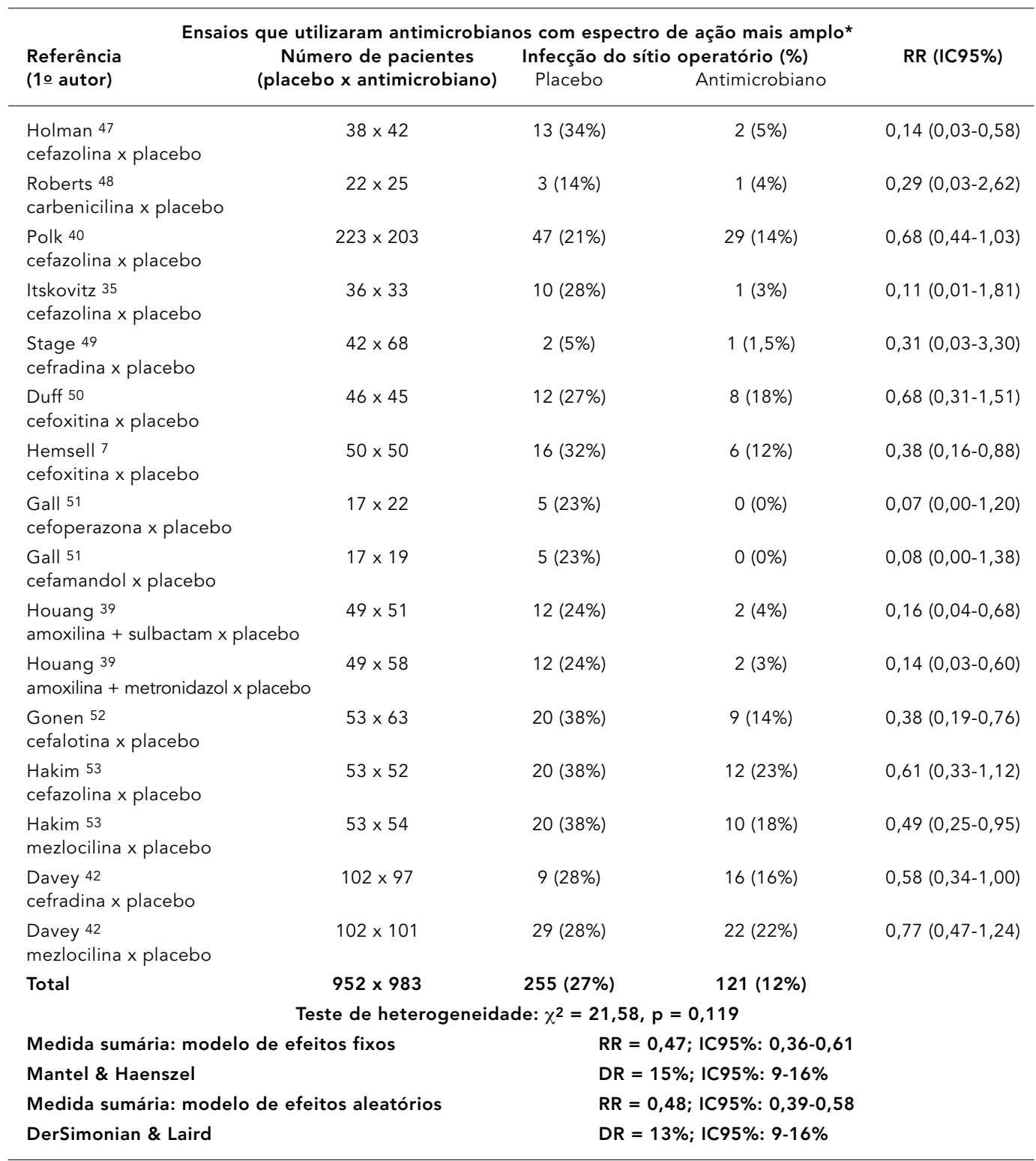

(continua)

carregado de cuidar do paciente até a alta e de proceder a avaliação (cegada) dos desfechos, o que implicaria que também o processo de cuidado pós-operatório tenha sido cegado, evitando viés em possíveis intervenções pós-randomização.

Também observou-se pobreza de informação nos ensaios sobre a estrutura de fatores prognósticos, o que comprometeu a verificação da existência ou não de variação de gravidade inter e intra ensaios. No entanto, foram incluí- dos no presente estudo somente ensaios com pacientes submetidas à histerectomia abdominal eletiva e não radical, e seguindo critérios de exclusão bastante semelhantes, o que contribui para a existência de relativa homogeneidade entre os ensaios quanto aos fatores prognósticos duração da cirurgia e comorbidades.

No presente trabalho, houve limitações à avaliação da execução e análise de dados dos estudos primários, já que a maior parte dos ensaios não relatou informações sobre perdas e 


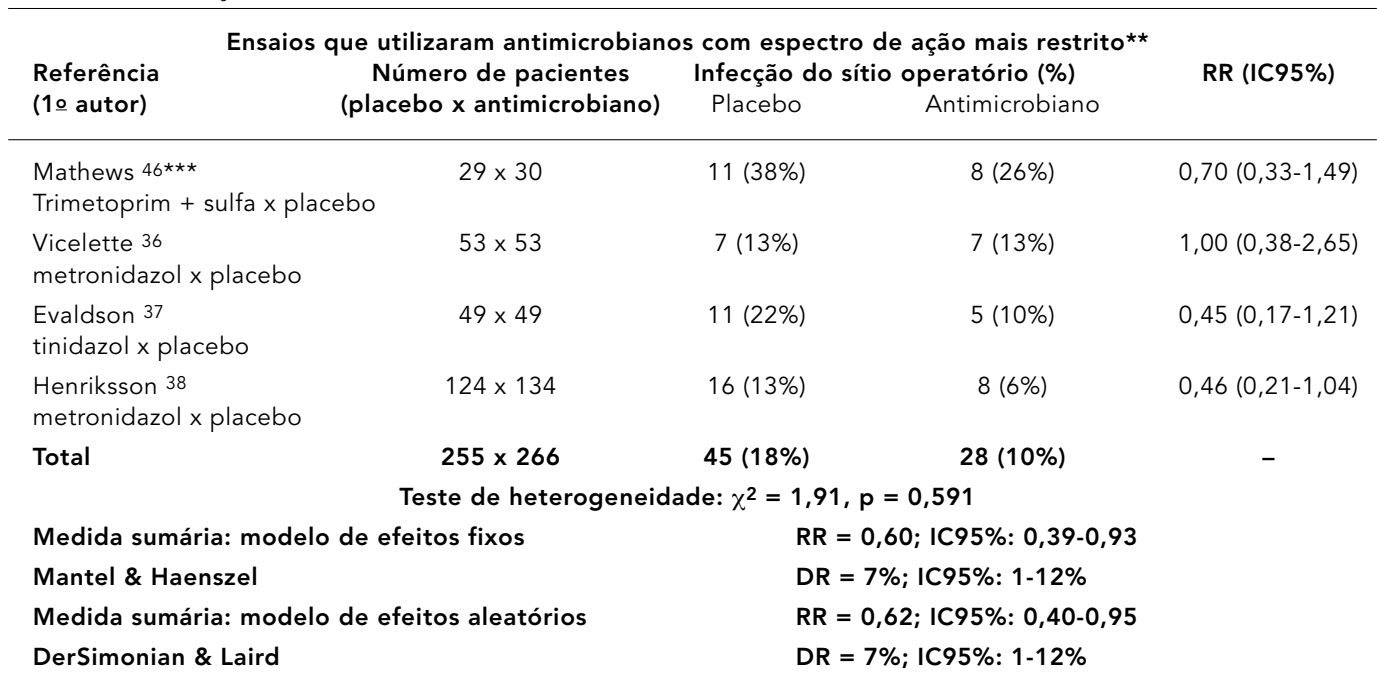

$\mathrm{RR}=$ risco relativo; $I C 95 \%$ = intervalo de confiança de 95\%; $\mathrm{DR}=$ diferença de risco.

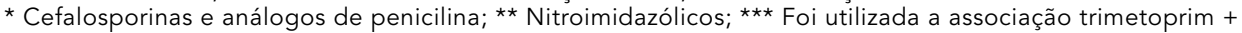

sulfametoxazol, classicamente utilizada no tratamento de infecção urinária, não em profilaxia cirúrgica.

razões para isso, ou sobre intervenções pósrandomização, inclusive cross-over e co-intervenções, nem se a análise realizada foi segundo intenção de tratar ou segundo tratamento efetivo. Aqui, também, dada a natureza eletiva da cirurgia, o baixo risco das pacientes em geral e a duração relativamente curta da intervenção e do seguimento, a ocorrência de perdas e de cross-over pode ter sido reduzida, conforme explicitado em alguns ensaios. Todavia, alguns estudos de tamanho médio apresentaram diferenças substantivas entre o número de pacientes tratados e o de controles. Além disso, três estudos revelaram perdas importantes, entre 10 e $25 \%$, pós-randomização. Por outro lado, embora os ensaios em geral tenham excluído da análise todas as perdas informadas, o cegamento do cirurgião pode ter protegido os ensaios de vieses na prescrição de intervenções pós-randomização.

As questões acima limitaram a avaliação da qualidade dos ensaios, deixando incerteza sobre a extensão do benefício.

Também houve nos ensaios diversidade quanto ao espectro de ação dos antimicrobianos. Por outro lado, a infecção pós-histerectomia do sítio cirúrgico pode ser causada por diversos tipos de bactérias, incluindo cocos aeróbios Gram-positivos, bacilos aeróbios Gramnegativos e anaeróbios 14. Essa diversidade de bactérias favoreceria em tese esquemas de amplo espectro.

Outra questão foi a variação de critérios usados para a medida dos desfechos nos en- saios, uma vez que foram freqüentemente utilizadas uma ou mais das alternativas de critérios diagnósticos compreendidas pelas definições abrangentes do CDC, a qual considera a evolução clínica da infecção e ainda a disponibilidade de recursos laboratoriais e de imagem 30,31. Por outro lado, medidas mais sensíveis também foram usadas, o que pode ter gerado variação nas diferenças de taxas entre os ensaios.

A despeito das possíveis falhas metodológicas apontadas, fontes potenciais de heterogeneidade 5,25 , o resultado do teste estatístico de heterogeneidade de resultados indicou homogeneidade $(p=0,199)$. Esse achado pode ser atribuído à baixa sensibilidade do teste 45 , mas também reflete o fato de os resultados dos ensaios selecionados serem positivos, via de regra (Figura 1).

\section{Conclusões}

A meta-análise do presente estudo fornece evidência de que a antibioticoprofilaxia, iniciada dentro de uma hora antes incisão, tem eficácia de cerca de $50 \%$ em evitar infecção do sítio operatório na histerectomia abdominal eletiva, não radical, com diferença de taxas de $10 \%$, aproximadamente. Por sua vez, as meta-análises publicadas, embora carentes de informações sobre o protocolo utilizado e sobre os ensaios por elas analisados, apontaram uma eficácia profilática semelhante. É possível que a homogeneidade dos estudos analisados tenha 
Taxas de infecção do sítio cirúrgico em ensaios placebo-controlados de antibioticoprofilaxia.

\begin{tabular}{|c|c|c|c|c|c|}
\hline \multirow{3}{*}{$\begin{array}{l}\text { Referência ( } 1 \text { o autor) } \\
\text { e comparação }\end{array}$} & \multicolumn{5}{|c|}{ Ensaios que registraram resultados separadamente* para os desfechos de infecção } \\
\hline & \multirow{2}{*}{$\begin{array}{l}\text { Número de pacientes } \\
\text { (placebo } \mathrm{x} \text { antimicrobiano) }\end{array}$} & \multicolumn{2}{|c|}{ Infecção da ferida operatória (\%) } & \multicolumn{2}{|c|}{ 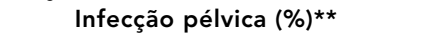 } \\
\hline & & Placebo & Antimicrobiano & Placebo & Antimicrobiano \\
\hline \multicolumn{6}{|l|}{ trimetropim + sulfa $\times$ placebo } \\
\hline \multicolumn{6}{|l|}{ cefalozina $x$ placebo } \\
\hline \multicolumn{6}{|l|}{ carbenicilina $\mathrm{x}$ placebo } \\
\hline \multicolumn{6}{|l|}{$\begin{array}{l}\text { Polk } 40 \\
\text { cefalozina x placebo }\end{array}$} \\
\hline \multicolumn{6}{|l|}{$\begin{array}{l}\text { Itskovitz } 35 \\
\text { cefalozina x placebo }\end{array}$} \\
\hline \multicolumn{6}{|l|}{ cefradina $x$ placebo } \\
\hline \multicolumn{6}{|l|}{$\begin{array}{l}\text { Duff } 50 \\
\text { cefoxitina } \times \text { placebo }\end{array}$} \\
\hline \multicolumn{6}{|l|}{$\begin{array}{l}\text { Vicelette } 36 \\
\text { metronidazol x placebo }\end{array}$} \\
\hline \multicolumn{6}{|l|}{$\begin{array}{l}\text { Hemsell } 7 \\
\text { cefoxitina } \times \text { placebo }\end{array}$} \\
\hline \multicolumn{6}{|l|}{$\begin{array}{l}\text { Gall } 51 \\
\text { cefoperazona x placebo }\end{array}$} \\
\hline \multicolumn{6}{|l|}{$\begin{array}{l}\text { Gall } 51 \\
\text { cefamandol x placebo }\end{array}$} \\
\hline \multicolumn{6}{|l|}{$\begin{array}{l}\text { Houang } 39 \\
\text { amoxilina + sulbactam } \times \text { placebo }\end{array}$} \\
\hline \multicolumn{6}{|l|}{$\begin{array}{l}\text { Houang } 39 \\
\text { amoxilina }+ \text { metronidazol } \times \text { placebo }\end{array}$} \\
\hline \multicolumn{6}{|l|}{$\begin{array}{l}\text { Gonen } 52 \\
\text { cefalotina } \times \text { placebo }\end{array}$} \\
\hline \multicolumn{6}{|l|}{$\begin{array}{l}\text { Evaldson } 37 \\
\text { tinidazol x placebo }\end{array}$} \\
\hline \multicolumn{6}{|l|}{$\begin{array}{l}\text { Hakim } 53 \\
\text { cefazolina x placebo }\end{array}$} \\
\hline \multicolumn{6}{|l|}{$\begin{array}{l}\text { Hakim } 53 \\
\text { mezlocilina } \times \text { placebo }\end{array}$} \\
\hline \multicolumn{6}{|l|}{$\begin{array}{l}\text { Henrikson } 38 \\
\text { metronidazol x placebo }\end{array}$} \\
\hline Total & $1.003 \times 1.051$ & $117(12 \%)$ & $54(5 \%)$ & $125(12 \%)$ & $57(5 \%)$ \\
\hline \multicolumn{2}{|l|}{ Teste de heterogeneidade } & \multicolumn{2}{|c|}{$\chi^{2}=13,31 ; p=0,715$} & \multicolumn{2}{|c|}{$\chi^{2}=11,26 ; p=0,843$} \\
\hline \multicolumn{2}{|c|}{ Medida sumária: modelo de efeitos fixos } & \multicolumn{2}{|c|}{$R R=0,46 ; I C 95 \%: 0,34-0,63$} & \multicolumn{2}{|c|}{$R R=0,46 ;$ IC95\%: 0,35-0,61 } \\
\hline \multicolumn{2}{|l|}{ Mantel \& Haenszel } & \multicolumn{2}{|c|}{$D R=4 \% ; I C 95 \%: 2-6 \%$} & \multicolumn{2}{|c|}{$D R=3 \% ; I C 95 \%: 1-4 \%$} \\
\hline \multicolumn{2}{|c|}{ Medida sumária: modelo de efeitos aleatórios } & $\mathbf{R} \mathbf{R}=0$ & $5 \%: 0,37-0,70$ & $\mathrm{RR}=$ & $5 \%: 0,37-0,66$ \\
\hline DerSimonian \& Laird & & 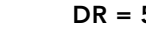 & $\%: 2-7 \%$ & $\mathrm{DR}=$ & $\%: 2-8 \%$ \\
\hline
\end{tabular}

$\mathrm{RR}=$ risco relativo; $I C 95 \%$ = intervalo de confiança de $95 \% ; \mathrm{DR}=$ diferença de risco.

* Foi excluído o ensaio de Davey et al. 41, que apresentou apenas um somatório dos resultados para infecção pélvica

e infecção da ferida operatória; ** Celulite pélvica, abscesso pélvico, infecção da cúpula vaginal. 
contribuído para esse achado, uma vez que parte dos ensaios da presente meta-análise constavam naquelas já existentes. Por outro lado, as revisões sistemáticas publicadas não apresentaram informação sobre ensaios comparativos. No presente estudo, a busca desses tipos de ensaios resultou no encontro de comparações entre esquemas de longa duração e com drogas de mesma geração, o que limitou a consecução de uma meta-análise de ensaios comparativos relevantes clinicamente.

A análise exploratória dose única versus dose múltipla não evidenciou maior eficácia para esquemas de doses múltiplas. Esse achado é relevante, considerando-se que o custo e a efetividade de esquemas mais prolongados podem modificar a relação custo-efetividade da antibioticoprofilaxia versus não intervenção ou dose única.

Conforme comentado na metodologia, o presente estudo utilizou dados relativos à CIM para produzir uma estimativa mais acurada do tempo de cobertura antimicrobiana. O número de ensaios com cobertura restrita ao período perioperatório, apenas três, foi menor que o de dose única, pois a meia-vida de alguns antimicrobianos usados em dose única era relativamente longa. $\mathrm{O}$ efeito sumário foi um pouco menor e o IC maior quando comparado ao do conjunto de ensaios e ao grupo de dose única. É questionável, todavia, a utilização da CIM, uma medida in vitro, para estimar o período de cobertura e conclusões in vivo, embora o caráter preventivo e a localização estudada não impliquem sítios de difícil penetração do antimicrobiano. Por outro lado, a CIM é utilizada para verificar se a droga pode ser eleita para tratamento, sendo assim discutível a sua utilização para inferir sobre intervenções profiláticas. Poder-se-ia argüir, usando o paradigma biológico, que uma concentração inibitória menor seja necessária para atingir uma eficácia profilática semelhante à terapêutica.

A escassez de estudos comparativos de antimicrobianos de boa qualidade, já comentada, limitou a análise da eficácia e a possibilidade de especificação de diretrizes quanto a diferentes classes e gerações de antimicrobianos relevantes para a nossa realidade, por exemplo, entre cefalosporinas de 1a e de $2 \underline{a}$ ou $3 \underline{a}$ gerações. Somente Hemsell et al. 34 apresentaram qualidade satisfatória, sendo comparadas duas cefalosporinas de gerações diferentes em regimes de dose única, encontrando-se eficácia marginalmente significativa. Portanto, a evidência disponível é insuficiente para inferirmos que existe vantagem em usar uma cefalosporina mais moderna frente àquela de 1ạ geração.
Foi possível apenas a comparação entre estudos placebo-controlados - de menor validade de natureza exploratória - que testaram antibióticos de diferentes espectros. Os resultados dos ensaios com antibióticos de espectro relativamente estreito (Tabela 4 ) indicam uma eficácia menor que a do conjunto dos ensaios, embora o número de ensaios e de pacientes nesse caso seja pequeno. Esses resultados sugerem que os antimicrobianos de largo espectro são um pouco mais eficazes que os de espectro estreito (Tabela 4), o que é consistente com o conhecimento sobre o espectro das bactérias associadas aos desfechos estudados, conforme referido anteriormente. Esses achados mereceriam ser testados por ensaios comparativos bem desenhados.

Por outro lado, a análise dos ensaios segundo subtipo de infecção (Tabela 5) mostrou efeitos sumários praticamente idênticos para os dois desfechos que foram semelhantes ao desfecho geral. Todavia, ela revela diferenças importantes: (a) a incidência de infecção da ferida operatória é muito maior que a de infecção pélvica em alguns ensaios, e o inverso também ocorre; (b) observa-se ainda diferenças de incidência para um mesmo desfecho entre os ensaios (heterogeneidade), o que também ocorreu com relação ao desfecho geral. A incidência diferenciada segundo desfecho (a) parece não ter sido devida, todavia, ao esquema profilático utilizado, podendo ter ocorrido ao acaso ou ter sido associada a diferenças nos critérios utilizados na delimitação dos desfechos. A diferença de eficácia entre ensaios (b), inclusive para o desfecho geral, pode ter sido devida ao acaso ou pode ter ocorrido devido a diferenças em co-intervenções, ou a falhas metodológicas, sobre as quais, todavia, os autores praticamente não informam.

Em resumo, a revisão efetuada fornece evidência a favor do uso da antibioticoprofilaxia na histerectomia abdominal eletiva, não radical. A análise dos ensaios não evidenciou maior eficácia para esquemas de doses múltiplas, o que é relevante economicamente. Ademais, embora estudos placebo-controlados sugiram eficácia superior para drogas de largo espectro, a evidência disponível com base em ensaios comparativos é insuficiente para inferir que existe vantagem em usar cefalosporinas de $3 \underline{a}$ geração frente àquelas de 1 a . 


\section{Resumo}

O presente estudo objetivou examinar a evidência científica disponível relativa à eficácia da antibioticoprofilaxia no caso da histerectomia, no sentido de subsidiar decisões relativas a financiamento e contribuir para a produção de diretrizes clínicas baseadas em evidências. Usando protocolo previamente elaborado, os ensaios disponíveis foram analisados quanto à pertinência e qualidade. A heterogeneidade clínica dos ensaios selecionados também foi examinada. O teste de heterogeneidade estatística sugeriu que os estudos são homogêneos, considerando-se o valor de $p>0,10$ como nível de significância para rejeitar a heterogeneidade. A combinação dos resultados dos 16 ensaios placebo-controlados selecionados resultou em razão de taxas sumárias de 0,49 (IC95\%: 0,41-0,59), ou seja, em eficácia de $51 \%$, e em diferença de taxas sumárias de 11\% (IC95\%: 8-14\%), utilizando-se o modelo de efeitos fixos. Os resultados observados usando-se o modelo de efeitos aleatórios foram muito semelhantes. A análise exploratória de esquemas de dose única versus doses múltiplas não indicou maior eficácia para esquemas de doses múltiplas. A evidência disponível, baseando-se em ensaios comparativos, é insuficiente para inferir que existe vantagem em usar cefalosporinas de terceira geração frente àquelas de primeira.

Meta-Análise; Antibioticoprofilaxia; Histerectomia; Medicina Baseada em Evidências

\section{Colaboradores}

Ambos os autores contribuíram na elaboração e revisão de todo o artigo.

\section{Referências}

1. Kluytmans J. Surgical infections including burns. In: Wenzel PR, editor. Prevention and control of nosocomial infection. Baltimore: Williams \& Wilkins; 1997. p. 841-65.

2. Ferraz EM, Ferraz AAB, Bacelar TS, D'Albuquerque HSD, Vasconcelos MDMM, Leão CS. Controle de infecção em cirurgia geral - resultados de um estudo prospectivo de 23 anos e 42.274 cirurgias. Rev Col Bras Cir 2001; 28:17-26.

3. Coordenação de Controle de Infecção Hospitalar, Ministério da Saúde. Consenso sobre o uso racional de antimicrobianos. Brasília: Coordenação de Controle de Infecção Hospitalar, Ministério da Saúde; 1998.

4. Petitti DB. Metanalysis, decision analysis, and cost-effectiveness analysis: methods for quantitative synthesis in medicine. New York: Oxford University Press; 2000.

5. Sacks HS, Berrier J, Reitman D, Ancona-Berk VA, Chalmers TC. Meta-analyses of randomized controlled trials. N Engl J Med 1987; 316:450-5.

6. Ministério da Saúde. Procedimentos hospitalares do SUS. http://www.datasus.gov.br/cgi/sih/pimap. htm (acessado em 01/Dez/2003).

7. Hemsell DL, Reisch J, Nobles B, Hemsell PG. Prevention of major infection after elective abdominal hysterectomy: individual determination required. Am J Obstet Gynecol 1983; 147:520-8.

8. Garibaldi RA, Cushing D, Lerer T. Risk factors for postoperative infection. Am J Med 1991; 91 Suppl 3B:158S-63S.

9. Haley RW, Culver DH, Morgan WM, White JW, Emori TG, Hooton TM. Identifying patients at high risk of surgical wound infection. A simple multivariate index of patient susceptibility and wound contamination. Am J Epidemiol 1985; 121:206-15

10. Shapiro M, Muñoz A, Tager IB, Schoenbaum SC, Polk BF. Risk factors for infection at the operative site after abdominal or vaginal hysterectomy. $\mathrm{N}$ Engl J Med 1982; 307:1661-6.

11. Soper DE, Bump RC, Hurt WG. Wound infection after abdominal hysterectomy: effect of the depth of subcutaneous tissue. Am J Obstet Gynecol 1995; 173:465-71

12. Persson E, Bergström M, Larsson P, Moberg P, Platz-Christensen JJ, Schedvins K, et al. Infections after hysterectomy. A prospective nation-wide Swedish study. The Study Group on Infectious Diseases in Obstetrics and Gynecology within the Swedish Society of Obstetrics and Gynecology. Acta Obstet Gynecol Scand 1996; 75:757-61.

13. Culver DH, Horan TC, Gaynes RP, Martone WJ, Jarvis WR, Emori TG, et al. Surgical wound infection rates by wound class, operative procedure and patient risk index. National Nosocomial Infections Surveillance System. Am J Med 1991; 91 Suppl 3B:152S-7S.

14. Mead PB. Postoperative infections. In: Thompson JD, Rock JA, editors. Te Linde's operative gynecology. 7th Ed. Philadelphia: Lippincott; 1992. p. 195207.

15. Burke JF. The effective period of preventive antibiotic action in experimental incisions and der- 
mal lesions. Surgery 1961; 50:161-8.

16. Shapiro M, Shimon D, Freund U, Sacks T. A decisive period in the antibiotic prophylaxis of cutaneous lesions caused by Bacteroides fragilis in guinea pigs. J Infect Dis 1980; 141:532.

17. Classen DC, Evans RS, Pestotnik SL, Horn SD, Menlove RL, Burke JP. The timing of prophylactic administration of antibiotics and the risk of surgical-wound infection. N Engl J Med 1992; 326: 281-6.

18. Stone HH, Hooper CA, Kolb LD, Geheber CE, Dawkins EJ. Antibiotic prophylaxis in gastric, biliary and colonic surgery. Ann Surg 1976; 184:44352.

19. Polk BF. Antimicrobial prophylaxis to prevent mixed bacterial infection. J Antimicrob Chemother 1981; 8 Suppl D:115-29.

20. Wttewaall-Evelaar EW. Meta-analysis of randomized controlled trials of antibiotic prophylaxis in abdominal hysterectomy. Pharm Weekbl Sci 1990; 12:296-9.

21. Mittendorf R, Aronson MP, Berry RE, Williams MA, Kupelnick B, Klickstein A, et al. Avoiding serious infections associated with abdominal hysterectomy: a meta-analysis of antibiotic prophylaxis. Am J Obstet Gynecol 1993; 169:1119-24.

22. Mittendorf R, Williams MA, Aronson MP, Berry RE, Kupelnick B, Chalmers TC. Use of prophylactic tinidazole to avoid the serious infections associated with total abdominal hysterectomy. Aust $\mathrm{N}$ Z J Obstet Gynaecol 1995; 35:314-5.

23. Fergusson D, Aaron SD, Guyatt G, Hébert P. Post_randomisation exclusions: the intention to treat principle and excluding patients from analysis. BMJ 2002; 325:652-4.

24. Lau J, Ioannidis JPA, Schmid CH. Quantitative synthesis in systematic review. Ann Intern Med 1997; 127:820-7.

25. Thompson SG. Why sources of heterogeneity in meta-analysis should be investigated. BMJ 1994; 309:1351-5.

26. Clarke-Pearson DL, Olt G, Rodriguez G, Boente M. Preopertative ealuation and postopertative management. In: Berek JS, Adashi EY, Hillard PA, editors. Novak's gynecology. Baltimore: William \& Wilkins; 1996. p. 543-617.

27. Dickersin K, Scherer R, Lefebvre C. Identifying relevant studies for systematic reviews. BMJ 1994; 309:1286-91.

28. Alderson P, Green S, Higgins J, editors. Reviewer's handbook [online]. http://www.cochrane.dk/ cochrane/handbook/hbook.htm (acessado em 21/Mai/2004).

29. Castro AA, Clark OAC, Atallah AN. Optimal search strategy for clinical trials in the Latin American and Caribbean Health Science Literature Database (LILACS): update. São Paulo Med J 1999; 117: 138-9.

30. Horan TC, Gaynes RP, Martone WJ, Jarvis WR, Emori TG. CDC definitions of nosocomial surgical site infections, 1992: a modification of CDC definitions of surgical wound infections. Infect Control Hosp Epidemiol 1992; 13:606-8.

31. Mangram AJ, Horan TC, Pearson ML, Silver LC, Jarvis WR. Guideline for prevention of surgical site infection, 1999. Hospital Infection Control
Practices Advisory Committee. Infect Control Hosp Epidemiol 1999; 20:250-78.

32. Periti P, Mazzei T, Orlandini F, Mini, E. Comparison of the antimicrobial prophylactic efficacy of cefotaxime and cephazolin in obstetric and gynaecological surgery. A randomised multicentre study. Drugs 1988; 35 Suppl 2:133-8.

33. Baldoni A, Cosco AG, Epicoco G, Affronti G, Giannone E, Gilardi G. Studio comparativo di chemioprofilassi antimicrobica a breve termine in chirurgia ginecologica: cefotetan versus cefazolina. Minerva Ginecol 1989; 41:149-55.

34. Hemsell DL, Johnson ER, Hemsell PG, Nobles BJ, Little BB, Heard MC. Cefazolin is inferior to cefotetan as single-dose prophylaxis for women undergoing elective total abdominal hysterectomy. Clin Infect Dis 1995; 20:677-84.

35. Itskovitz J, Fisher M, Urbach J, Brandes JM. The effect of a short-term course of antibiotic prophylaxis on patients undergoing total abdominal hysterectomy. Eur J Obstet Gynecol Reprod Biol 1980; 11:101-7.

36. Vincelette J, Finkelstein F, Aoky FY, Ogilvie RI, Richards GK, Seymour RJ. Double-blind trial of perioperative intravenous metronidazole prophylaxis for abdominal hysterectomy. CMAJ 1982; 127:119-23.

37. Evaldson GR, Lindgren S, Malmborg A, Nord CE. Single-dose intravenous tinidazole prophylaxis in abdominal histerectomy. Acta Obstet Gynecol Scand 1986; 65:361-5.

38. Henriksson L, Colling-Salting A, Frik G, Kullander S, Sandholm L, Ursing J, et al. Metronidazole prophylaxis to prevent infections after total abdominal hysterectomy. Acta Obstet Gynecol Scand 1998; 77:116-9.

39. Houang ET, Watson C, Howell R, Chapman M. Ampicilin combined with sulbactam or metronidazole for single-dose chemoprophylaxis in major gynaecological surgery. J Antimicrob Chemother 1984; 14:529-35.

40. Polk BF, Tager IB, Shapiro M, Goren-White B, Goldstein P, Schoenbaum SC. Randomized clinical trial of perioperative cefazolin in preventing infection after hysterectomy. Lancet 1980; 1:437-40.

41. Lau J. Meta-analyst: a program for the meta-analysis of 2 by 2 tables. Version 0,991. Boston: New England Medical Center; 1997.

42. Davey PG, Duncan ID, Edward D, Scott AC. Costbenefit analysis of cephradine and mezlocillin prophylaxis for abdominal and vaginal hysterectomy. Br J Obstet Gynaecol 1988; 95:1170-7.

43. Egger M, Smith S, Schneider M, Minder C. Bias in meta-analysis detected by a simple, graphical test. BMJ 1997; 315:629-34.

44. Clarke M, Oxman AD. Cochrane reviewers' handbook 4.1.4. (Cochrane Review). In: The Cochrane Library, Issue 4, 2001. Oxford: Update Software.

45. Thompson SG, Pocock SJ. Can meta-analyses be trusted? Lancet 1991; 338:1127-30.

46. Mathews DD, Ross H, Cooper J. A douple-blind trial of single-dose chemoprophylaxis with cotrimoxazole during total abdominal hysterectomy. Br J Obstet Gynaecol 1977; 84:894-7.

47. Holman JF, McGowan JE, Thompson JD. Perioperative antibiotics in major elective gynecologic 
surgery. South Med J 1978; 71:417-20.

48. Roberts JM, Homesley HD. Low-dose carbenicilin prophylaxis for vaginal and abdominal hysterectomy. Obstet Gynecol 1978; 52:83-7.

49. Stage AH, Glover DD, Vaugham JE. Low-dose cephradine prophylaxis in obstetrics and gynecologic surgery. J Reprod Med 1982; 27:113-9.

50. Duff P. Antibiotic prophylaxis for abdominal hysterectomy. Obstet Gynecol 1982; 60:25-9.

51. Gall AS, Hill GB. Cefoperazone as a prophylactic agent in abdominal hysterectomy. Rev Infect Dis 1983; 5 Suppl:200-1.

52. Gonen R, Hakin M, Samberg I, Levitan Z, Sharf LM. Short-term prophylactic antibiotic for elective abdominal hysterectomy: how short? Eur J Obstet Gynecol Reprod Biol 1985; 20:229-34.

53. Hakim M, Gonen R, Levitan Z, Sharf M. Broad spectrum antibiotics as short term prophylaxis for elective abdominal hysterectomy: comparison of mezlocillin, cefazolin and placebo. Int J Gynaecol Obstet 1986; 24:157-60.

Apresentado em 16/Dez/2004

Versão final reapresentada em 01/Jun/2004

Aprovado em 09/Jul/2004 